\title{
Guía de práctica clínica para el tratamiento de la diabetes mellitus tipo 2: manejo inicial
}

\section{Clinical Practice Guideline for Treatment of Type 2 Diabetes} Mellitus: Initial Treatment

Fecha de recepción: 27/09/2016 | Fecha de aprobación: 18/01/2017

Pablo Aschner M.

Hospital Universitario San Ignacio, Colombia

Óscar Mauricio Muñoz V. ${ }^{a}$

Hospital Universitario San Ignacio, Colombia

Diana Girón C.

Pontificia Universidad Javeriana, Colombia

Olga Milena García M.

Hospital Universitario San Ignacio, Colombia Daniel Gerardo Fernández Á.

Hospital Universitario San Ignacio, Colombia Luz Ángela Casas

Universidad del Valle, Colombia

Luisa FERnANDA BOHÓRQuez

Universidad Nacional de Colombia, Colombia

Clara María Arango T.

Universidad de Antioquia, Colombia

Liliana Carvajal

Asociación Colombiana de Diabetes, Colombia

Doris Amanda RamíRez

Universidad Nacional de Colombia, Colombia

a Autor de correspondencia. Correo

electrónico: o.munoz@javeriana.edu.co

* Representante de la población blanco

** Representante de la población blanco

Cómo citar: Aschner P, Muñoz OM, Girón D, García OM, Fernández DG, Casas LA, Bohórquez LF, Arango CM, Carvajal L, Ramírez DA, Sarmiento JG, Colón CA, Correa NF, Alarcón P, Bustamante ÁA, Fino V, Penagos E. Guía de práctica clínica para el tratamiento de la diabetes mellitus tipo 2: manejo inicial. Univ Med. 2017;58(4):1-13. doi: https://doi.org/10.1 1144/ Javeriana.umed58-4.dmmi
Juan Guillermo Sarmiento Hospital Universitario San Ignacio, Colombia

Cristian Alejandro Colon Hospital Universitario San Ignacio, Colombia

Néstor Fabián Correa G. Pontificia Universidad Javeriana, Colombia Pilar Alarcón R.

Pontificia Universidad Javeriana, Colombia Álvaro Andrés Bustamante S. Pontificia Universidad Javeriana, Colombia Víctor Fino* ERnesto Penagos**

\section{RESUMEN}

Introducción: En la actualidad es ampliamente aceptada la metformina como manejo farmacológico inicial para el tratamiento de la diabetes mellitus tipo 2 (DMT2). Resulta, sin embargo, controversial si en algunos tipos de pacientes puede iniciarse tratamiento únicamente con cambios intensivos de estilo de vida o si existen grupos en quienes debería 
iniciarse desde el inicio terapia farmacológica combinada. Objetivo: Definir el impacto de estrategias de cambio intensivo en la dieta y de ejercicio, así como de la terapia farmacológica con metformina asociada a un segundo antidiabético oral como estrategias de manejo inicial en pacientes con DMT2 recién diagnosticada. Métodos: Se elaboró la guía de práctica clínica, siguiendo los lineamientos de la guía metodológica del Ministerio de Salud y Protección Social colombiano. Se revisó la evidencia disponible de forma sistemática y se formularon las recomendaciones utilizando la metodología GRADE. Conclusiones: En pacientes con DMT2 recién diagnosticada no se recomienda el manejo únicamente con cambios intensivos del estilo de vida; estos deben acompañar el manejo farmacológico con metformina, dando prelación a los componentes de la dieta mediterránea y al ejercicio aeróbico. En los pacientes con DMT2 recién diagnosticada y niveles de $\mathrm{HbA} 1 \mathrm{C}>8 \%$ se recomienda utilizar terapia combinada desde el inicio con metformina y otro antidiabético oral, siendo de primera elección los inhibidores de DPP-4.

Palabras clave

diabetes mellitus tipo 2; metformina; tratamiento primario; guías de práctica clínica.

\begin{abstract}
Introduction: Today, metformin is widely accepted as standard initial pharmacologic treatment for diabetes mellitus type 2 (DMT2). However, it is controversial if in some groups of patients, the treatment can be initiated only with life style changes, or if there are groups who should begin with combined therapy since the beginning. Aim: To define the effect of intensive strategies for change of diet or exercise, and the effect of combined therapy with metformin and a second oral antidiabetic, as initial treatment in patients with newly DMT2. Methods: A clinical practice guide has been developed following the broad outline of the methodological guide from the Colombian Ministry of Health and Social Welfare. with the aim of systematically gathering scientific evidence and formulating recommendations using the GRADE (Grading of Recommendations Assessment, Development and Evaluation) methodology. Conclusions: In patients with recently diagnosed DMT2, initial treatment with lifestyle changes only is not recommended. However, it is recommended that lifestyles changes must begin simultaneously with metformin, including the components of a Mediterranean diet and aerobic exercise. In patients with recently diagnosed DMT2 and HbA1c levels $>8 \%$, it is recommended to administer a combined therapy from the beginning with metformin and another oral antidiabetic medication. The DPP4 inhibitor is recommended.
\end{abstract}

Keywords

type 2 diabetes mellitus; metformin; primary treatment; clinical practice guidelines.

\section{Introducción}

El UK Prospective Diabetes Study (UKPDS) ha sido el estudio más grande y de mayor seguimiento en personas con diabetes mellitus tipo 2 (DMT2) y se diseñó para establecer la efectividad del control glucémico intensivo inicial en las complicaciones de la diabetes [1]. El manejo intensivo se inició con sulfonilurea o insulina basal, y el convencional, solamente con dieta. A pesar de que el control glucémico se deterioró en ambos grupos analizados en dicho estudio, se logró mantener una diferencia de la prueba de hemoglobina glucosilada (HbA1c) en el 0,9\%; mientras que el grupo intensivo mantuvo una mediana de $\mathrm{HbA} 1 \mathrm{c}$ del $7 \%$, con lo cual se redujo significativamente la incidencia de cualquier complicación relacionada con la diabetes, en particular la incidencia de complicaciones microvasculares.

En un subgrupo de pacientes con sobrepeso se inició el manejo intensivo con metformina, y a pesar de que mantuvo una mediana de HbA1c un poco más alta $(7,4 \%)$ y de que la diferencia con el grupo control fue apenas del 0,6\%, este grupo tuvo la mayor reducción de la incidencia de cualquier complicación relacionada con la diabetes y una reducción significativa de mortalidad, con baja incidencia de hipoglucemia. El peso en ese grupo de pacientes se mantuvo muy similar al del grupo control [2]. A partir de estos resultados, las guías de práctica clínica vienen proponiendo el control glucémico intensivo (HbA1c del $7 \%$ ), iniciando el tratamiento preferiblemente con metformina $[3,4,5,6]$.

Adicionalmente, las guías reconocen el papel fundamental de los cambios en el estilo de vida como parte del tratamiento de la DMT2 $[3,4,5]$. Los cambios en la actividad física y el adecuado manejo nutricional favorecen un control metabólico permanente, además de la normalización y el mantenimiento del peso, lo que en últimas llevará a disminuir el riesgo de enfermedad cardiovascular [7,8].

El manejo nutricional de los pacientes con DMT2 debe incluir una dieta estructurada con determinadas características que ayuden a cada 
paciente. Dentro de las dietas que han sido evaluadas formalmente se encuentran las dietas reducidas en calorías (1000 a $2000 \mathrm{kcal}$ diarias) con remplazo a base de soya, dietas bajas en carbohidratos $(<45 \%$ del consumo calórico total), dietas bajas en grasas $(<30 \%$ del consumo calórico toral y $<10 \%$ de la ingesta de grasa saturada) incluyendo dietas veganas, dietas con índice glucémico bajo y dieta mediterránea. Esta última es la que ha recibido más atención y ha sido más ampliamente estudiada [9] (tabla 1).

Tabla 1. Recomendaciones dietéticas de los participantes del estudio Prevención con Dieta Mediterránea

\begin{tabular}{|c|c|}
\hline Tipo de alimento & Porción/día \\
\hline \multicolumn{2}{|c|}{ Recomendado } \\
\hline Aceite de oliva* & Más o 4 cucharas al día \\
\hline Frutos secos/maní & Más o 3 porciones a la semana \\
\hline Frutas frescos & Más o 3 porciones al día \\
\hline Vegetales & Más o 2 porciones al día. \\
\hline Pescado/mariscos & Más o 3 porciones a la semana \\
\hline Legumbres & Más o 3 porciones a la semana \\
\hline Sofrito** & Más o 3 porciones a la semana \\
\hline Came blanca & $\begin{array}{l}\text { Consumir en lugar de carne } \\
\text { roja }\end{array}$ \\
\hline Vino con las comidas & $\begin{array}{l}7 \text { copas a la semana con las } \\
\text { comidas }\end{array}$ \\
\hline \multicolumn{2}{|r|}{ Evitar } \\
\hline Gaseosas & Menos de una bebida al día \\
\hline $\begin{array}{l}\text { Productos de } \\
\text { panadería }\end{array}$ & $\begin{array}{l}\text { Menos de } 3 \text { porciones a la } \\
\text { semana. }\end{array}$ \\
\hline Grasas & Menos de una porción al día \\
\hline $\begin{array}{l}\text { Carnes rojas y } \\
\text { procesadas }\end{array}$ & Menos de una porción al día \\
\hline
\end{tabular}

* La cantidad de aceite incluye para cocinar, para las ensaladas y cualquier otro tipo de alimento. El objetivo del estudio fue una porción diaria de 50 gramos o más por día de aceite.

**Es una salsa hecha de tomate y cebolla mezclado con hierbas aromáticas y ajo, cocinado a fuego lento con aceite de oliva. Fuente: tomada del estudio Predimed [9].

Por otro lado, existen diferentes tipos de actividad física que formalmente se han evaluado como parte del manejo de la DMT2: el ejercicio aeróbico (por ejemplo, caminar, bicicleta, trotar, entre otros), el ejercicio de resistencia (por ejemplo, ejercicios cortos, repetitivos con peso, bandas de resistencia o ejercicios que incrementen el estiramiento o fortalezcan la masa muscular) o combinaciones de ellos. Todos mejoran el control glucémico, independientemente de la reducción del peso corporal $[10,11,12]$. Sin embargo, resulta discutido si el manejo inicial, incluyendo exclusivamente cambios en el estilo de vida, puede ser adecuado y suficiente en pacientes con DMT2 recién diagnosticada [13].

Entre aquellos pacientes que en el momento del diagnóstico la HbA1c es mayor de $8,5 \%$ una opción interesante es iniciar con dos medicamentos para controlar la glucemia [6,14], considerando que el uso de un medicamento antihiperglucémico como monoterapia disminuye las concentraciones de HbAlc entre el 0,5\% y el 1,5\%, aunque insuficiente para estos pacientes. Además, el uso de terapia combinada desde el inicio puede traer múltiples beneficios: primero, puede ayudar a preservar la función de la célula beta $y$, por consiguiente, mantener el control glucémico a largo plazo [15,16]. Segundo, la terapia combinada no resulta en mayores efectos adversos asociados con los medicamentos, porque en la terapia combinada no se requiere llegar a las dosis máximas de los medicamentos [17,18,19]. Tercero, muchos médicos, independientemente de su especialidad, no evalúan de forma sistemática si se está llegando a las metas de manejo; por tanto, no aumentan la dosis del medicamento antihiperglucémico y dejan que la historia natural de la enfermedad prosiga. Esto ha sido denominado inercia clínica, situación documentada en varios estudios [20].

Es importante aclarar que al iniciar dos medicamentos antihiperglucemiantes, deben usarse dos tipos que no compartan mecanismo de acción. Los que se han usado como terapia combinada inicial asociados a metformina incluyen: sulfonilureas, tiazolidinedionas, inhibidores de dipeptidil peptidasa 4 (DPP4) e inhibidores del cotransportador sodio-glucosa (SGLT2).

El Ministerio de Salud y Protección Social encargó a la Pontificia Universidad Javeriana y a la Alianza del Centro Nacional de Investigación en Evidencia y Tecnologías en Salud (Cinets), conformada adicionalmente por la Universidad Nacional de Colombia y la Universidad de Antioquia, el desarrollo de una Guía clínica sobre la prevención, detección temprana, diagnóstico, 
tratamiento y seguimiento de la diabetes mellitus en la población mayor de 18 años. El resultado de ese proceso, en lo referente al tratamiento inicial, se presenta de forma resumida en el presente artículo, que incluye la evidencia que evalúa el uso de intervenciones intensivas de estilo de vida como manejo inicial único en pacientes con diabetes mellitus recién diagnosticada. Adicionalmente, se presenta la evidencia que apoya la terapia combinada como tratamiento inicial de la DMT2 y propone grupos en los que esta terapia podría recomendarse. Documentos complementarios abordarán los temas de falla al tratamiento de primera línea y metas de manejo.

Cabe resaltar que en este proceso también participaron activamente la Asociación Colombiana de Endocrinología, así como la Asociación Colombiana de Diabetes y el Cinets.

\section{Métodos}

El proceso de desarrollo de la guía se describe detalladamente en el Manual para el desarrollo de guías de práctica clínica y en la actualización de dicha metodología (disponible en la página web del Ministerio de Salud y Protección Social: http ://gpc.minsalud.gov.co) [21].

El desarrollo de la guía partió de preguntas específicas seleccionadas por su importancia clínica. Se les dio prioridad a los temas en que se consideró aún existe una discusión activa en la literatura mundial y a aquellos temas en los que se detectó una variabilidad injustificada en las conductas realizadas por los profesionales de salud en Colombia. Cabe aclarar que se aceptaron como premisas a la presente guía aquellos temas ampliamente aceptados en la literatura mundial y que no ameritan, por tanto, un nuevo análisis de la literatura científica, y como tal se presentan en el presente documento (por ejemplo, el uso de metformina en monoterapia como primera línea de manejo farmacológico en pacientes con DMT2 recién diagnosticada y concentraciones de HBA1c menores al $8 \%$ ).

La metodología planteada garantiza una búsqueda sistemática de la evidencia científica (incluyendo tanto revisiones sistemáticas de la literatura como estudios primarios). Así mismo, establece de forma clara los criterios para la selección de la evidencia que se va a utilizar, evaluando cuidadosamente su calidad. La versión completa de la guía, en la que se presentan los resultados de todas estas evaluaciones, pueden consultarse en la página web del Ministerio de Salud y Protección Social colombianos, así como en la página web de la Alianza Cinets.

El grupo metodológico preparó un resumen de la evidencia disponible y lo presentó al panel completo durante las reuniones de generación de recomendaciones. Este panel incluyó representantes de diversas especialidades médicas (endocrinólogos, médicos internistas, médicos generales), así como otros profesionales de la salud (nutricionistas, terapeutas físicos, entre otros). Cabe resaltar que durante todo el proceso se contó también con representantes de los pacientes, que aportaron activamente en la generación de recomendaciones. Todos los integrantes del panel presentaron abiertamente la declaración de sus conflictos de interés. Estos documentos están disponibles en la versión completa de la guía.

Durante las reuniones de generación de recomendaciones se siguió la metodología propuesta por el Grading of Recommendations Assessment, Development and Evaluation Working Group (GRADE), que permite tener en cuenta no solo la calidad de la evidencia disponible, sino consideraciones de costos, preferencias de los pacientes y la relación entre los beneficios y riesgos de las tecnologías de interés (pruebas, estrategias de manejo, intervenciones o medicamentos). Las recomendaciones presentadas muestran, por tanto, la fuerza de la recomendación (fuerte o débil), su dirección (a favor o en contra de la intervención), así como la calidad de la evidencia que la respalda (muy baja, baja, moderada, alta o consenso de expertos).

La versión final de la guía fue evaluada por pares nacionales, seleccionados por el Ministerio de Salud y de la Protección Social colombiano, expertos tanto en DMT2 como en metodología. 
Sus aportes fueron tenidos en cuenta por el grupo desarrollador de la guía.

\section{Resultados}

\section{Cambios intensivos del estilo de vida}

La búsqueda sistemática de la literatura nos permitió identificar la evidencia disponible acerca del efecto de programas de ejercicio asociados a cambios dietarios realizados de forma simultánea dentro de un programa estructurado. Un estudio reciente de alta calidad metodológica evaluó el impacto de una intervención multifactorial con dieta y ejercicios intensivos, con manejo usual y educación en diabetes, dirigida a perder peso, en pacientes con DMT2 de mediana duración alrededor de cinco años [22]. La dieta incluía consumo de 1200 a $1800 \mathrm{kcal}$ por día (con < 30\% de calorías provenientes de la grasa y $>15 \%$ de las proteínas) y el uso de productos para el remplazo de comidas. La actividad física debía ser de moderada intensidad con duración mínima de 175 minutos a la semana. El grupo de comparación recibió manejo usual y educación en diabetes.

A pesar de que los pacientes del programa lograron perder un $8,6 \%$ del peso corporal al cabo del primer año y un $6 \%$ al final del estudio (vs. 0,7 y 3,5\% en el grupo control) y de un leve cambio en la HbA1c, no se logró una reducción significativa de la incidencia de eventos cardiovasculares fatales o no fatales y el estudio se suspendió prematuramente por los resultados del análisis de futilidad. Específicamente, no se evidenciaron cambios en la frecuencia de muerte de cualquier causa (HR: 0,85; IC95 \%: 0,69-1,04), muerte cardiovascular (HR: 0,88; IC95\%: 0,61-1,29), infarto agudo de miocardio (HR: 0,84; IC95\%: 0,68-1,04) o ataque cerebrovascular (HR: 1,05; IC95 $\%$ : 0,77-1,42). Es importante anotar que los beneficios iniciales del cambio intensivo del estilo de vida (pérdida de peso, disminución de la HbA1c) comenzó a perderse a partir del primer año.
En todo caso, es indudable que los cambios en el estilo de vida se deben iniciar e intensificar en el momento del diagnóstico, preferiblemente dentro de un programa estructurado. A continuación se presenta la evidencia disponible en lo referente a intervenciones dietarias y ejercicio de forma individual.

\section{Manejo dietario}

Como parte del manejo de la DMT2 se han evaluado diferentes tipos de dieta. La información más relevante proviene del estudio Prevención con Dieta Mediterránea (Predimec) [9], de cohortes observacional multicéntrico aleatorizado, que evaluó los efectos de las dieta mediterránea suplementada con aceite de oliva extravirgen o suplementada con nueces, comparada con una dieta control baja en grasas, sobre el riesgo cardiovascular. Los resultados sugieren un impacto positivo en la incidencia de un punto final compuesto que incluía ataque cerebrovascular, infarto agudo de miocardio y sobremortalidad (HR: 0,70; IC95 \%: 0,53-0,91). Cabe aclarar que este estudio de cohortes consideró la inclusión de pacientes al estudio que cumplieran o no el criterio diagnóstico de DMT2, por lo que la evidencia que aporta al presente trabajo es indirecta.

Otras intervenciones nutricionales evaluadas incluyen las dietas reducidas en calorías con remplazo a base de soya. Una revisión sistemática [23] demostró que no hay cambios clínica ni estadísticamente significativos en las concentraciones de $\mathrm{HbAlc}$ (diferencia de medias: $-0,09 \%$; IC95\%: $-0,50-0,31)$. Resultados similares fueron reportados en una revisión sistemática [24] que evaluó los efectos de las dietas muy bajas en calorías (400-500 $\mathrm{kcal} /$ día) comparadas con dietas bajas en calorías (1000-2000 kcal/día), y en otra que evaluó las dietas bajas en grasa (incluyendo dietas veganas) [25]. (Diferencia de medias: -0,20\%; IC95\%: $-0,71-0,30)$.

Una revisión sistemática [26] evaluó el efecto las dietas bajas en carbohidratos comparado con otras dietas (dietas individualizadas por 
la Asociación Americana de Diabetes). El resultado mostró una disminución de $\mathrm{HbA1c}$ estadísticamente significativa; pero mínima desde el punto de vista clínico para el grupo de dietas bajas en carbohidratos (diferencia de medias: -0,12\%; IC95\%: -0,24-0,00; $\mathrm{p}=0,04)$. No se presentó una diferencia estadísticamente significativa en el cambio de peso corporal (diferencia de medias: $-0,69 \mathrm{~kg}$; IC95 \%: - 1,77-0,39 kg).

$\mathrm{Al}$ evaluar las dietas de bajo índice glucémico, un metanálisis [27] encontró disminución de la HbA1c (diferencia de medias: $-0,4 \%$; IC95 $\%:-0,7 \%-0,2 ; p=0,001)$. Sin embargo, una segunda revisión sistemática no encontró diferencias significativas [26] en el control glucémico. Los diferentes resultados pueden estar dados por el grupo de comparación que fue dietas de alto índice glucémico en el primero y dietas recomendadas por la Asociación Americana de Diabetes, para el segundo.

Ninguna de las revisiones sistemáticas, ni de los estudios de control aleatorios identificados brinda información respecto al efecto de las dietas mencionadas sobre eventos macrovasculares, microvasculares y mortalidad.

\section{Ejercicio físico}

Al evaluar el impacto de la actividad física, la evidencia demuestra que el ejercicio aeróbico está inversamente asociado con mortalidad por cualquier causa y por causa cardiovascular [28]. Este estudio evaluó los resultados de 12 cohortes de sujetos con DMT2 seguidos durante un promedio de 9,4 años y encontró que pertenecer al grupo de actividad física moderada y al grupo de mayor actividad física durante el tiempo de ocio tienen el mayor impacto en reducción de mortalidad (HR: 0,62; IC95 \%: 0,49-0,78 y HR: 0,73; IC95 \%: 0,57-0,93, respectivamente). El riesgo de mortalidad cardiovascular se redujo en el grupo de los que caminaban dos a cuatro y media horas por semana (HR 0,54; IC95 \%: 0,36-0,82).

Una revisión sistemática [29] evaluó el efecto del ejercicio aeróbico comparado con consejería para realizar actividad física en el control glucémico. Se encontró una reducción significativa en las cantidades de $\mathrm{HbA1c}(-0,73$ \%; IC95 \%: $-1,06 \%$ a $-0,40 \%)$. Una segunda revisión sistemática de la literatura del mismo autor [30] demostró cómo a mayor frecuencia de sesiones de ejercicio aeróbico a la semana se presentaban mayores reducciones en las concentraciones de $\mathrm{HbA1c}$; también se encontró una disminución del 0,39\% adicional por cada sesión de ejercicio. Cabe recalcar que solamente aconsejar actividad física no tiene un efecto significativo en la $\mathrm{HbA1c}$.

Por último, una nueva revisión sistemática [29] evaluó el efecto del ejercicio de resistencia en la HbA1c, comparado con consejería para realizar actividad física. Se encontró una reducción significativa (diferencia de medias: 0,57 \%; IC95 \%: - 1,14\% a -0,01\%).

Ninguna de las revisiones sistemáticas ni de los estudios de caso identificados brinda información respecto al impacto de programas de ejercicio aérobico o ejercicio de resistencia estructurado en la incidencia de mortalidad, eventos macrovasculares, eventos microvasculares o hipoglucemia.

Tratamiento farmacológico combinado desde el inicio

Se han evaluado diferentes medicamentos en combinación con metformina, como manejo farmacológico inicial en pacientes con DMT2. Entre ellos se encuentran sulfonilureas, tiazolidinedionas, gliptinas y SGLT2. Una revisión sistemática [15] comparó las combinaciones con la metformina como monoterapia. Todos los medicamentos evaluados presentaron disminuciones de la $\mathrm{HbAlc}$ significativas y de similar magnitud (DM: $-0,43 \%$; IC95\%: $-0,56$ a $-0,30)$ [15]. De igual forma, todos los antidiabéticos orales aumentaron de forma significativa la probabilidad de alcanzar metas de control glucémico $<7 \%$ de HbA1c (RR: 1,40; IC95 \%: $1,33-1,48)$. 
Cabe resaltar que existe evidencia de baja calidad que sugiere una disminución del riesgo de mortalidad e infarto agudo del miocardio asociado al uso de DPP4 [31] y pioglitazona [32], y que ninguna de las terapias combinadas sugiere un impacto en el riesgo de complicaciones microvasculares.

Dada la similitud en la eficacia de los diferentes medicamentos, resulta fundamental evaluar los riesgos de efectos adversos para definir la mejor opción. En ese sentido, la evidencia demuestra que la terapia combinada desde el inicio, incluyendo un segundo antidiabético oral como inhibidores DPP4 [33], pioglitazona [34] o SGLT2 [35], no se asocia con aumento del riesgo de hipoglucemia, al comparar con monoterapia con metformina. Por el contrario, la terapia combinada con sulfonilureas, desde el inicio, se asocia con un incremento estadística y clínicamente significativo del riesgo de hipoglucemia, siendo mayor cuando la combinación incluye glibenclamida (RR: 16,05; IC95 \%: 6,22-41,39), que cuando se combina con glimepirida o glicazida (RR: 2,08; IC95\%: 0,74-5,86) [36].

Desde el inicio, la terapia combinada que incluyó pioglitazona [34] o sulfonilureas [15] fue asociada con aumento del peso, comparado con la disminución de peso en pacientes que recibieron monoterapia con metformina, o la terapia combinada desde el inicio incluyendo DPP4 [33] o SGLT2 [35].

Adicionalmente, la evidencia sugiere un incremento del riesgo de falla cardiaca (HR: 1,41; IC95 \%: 1,14-1,76) [37], riesgo de fracturas (OR: 1,45; IC95 \%: 1,18-1,79) [38] y de cáncer de vejiga (HR: 1,23; IC95 \%: 1,09-1,39) [39] asociado al uso de pioglitazona. Así mismo, la evidencia sugiere que el uso de SGLT2 se asocia con aumento de riesgo de infecciones urinarias y de infecciones del tracto genital (vulvovaginitis) [35].

\section{Conclusión}

De acuerdo con la evidencia presentada, el efecto del ejercicio aeróbico o del ejercicio de resistencia asociado a cambios dietarios realizados de forma simultánea dentro de un programa estructurado no tiene un impacto significativo en la incidencia de mortalidad o eventos micro o macrovasculares; adicionalmente, el impacto en el control glucémico y el peso son moderados y tienden a perderse con el paso del tiempo. Por lo tanto, no parece razonable recomendar los cambios intensivos de estilo de vida como manejo único en pacientes con diabetes mellitus recién diagnosticada.

Sin embargo, resultan innegables los beneficios de las intervenciones dietarias y el ejercicio como parte de un manejo integral que incluya manejo farmacológico preferiblemente con metformina.

En lo referente a las intervenciones nutricionales, solo la dieta mediterránea incide positivamente frente a ataques cerebrovasculares, infarto agudo de miocardio y sobremortalidad. Ninguna de las otras dietas evaluadas (de remplazo a base de soya, bajas en grasas, muy bajas en grasa, bajas en carbohidratos o dieta con índice glucémico bajo) evidenciaron cambios clínicamente significativos sobre el control glucémico y la reducción del peso posterior al año de seguimiento; tampoco impacto en los desenlaces micro y macrovasculares, calidad de vida e hipoglucemia.

Las dietas bajas en calorías, bajas en carbohidratos y con índice glucémico bajo muestran reducciones de $\mathrm{HbA1c}$ con significancia estadística; sin embargo, tienen una pobre significancia clínica. La disminución del peso de todas las dietas resulta con pobre significancia clínica, a excepción de la observada en las dietas bajas en calorías.

La evidencia encontrada demuestra que el riesgo de mortalidad se reduce de forma tanto clínica como estadísticamente significativa, al comparar a las personas con alto nivel de actividad física de tipo aeróbico (caminata) con aquellas con bajos índices de actividad, incluso en aquellos en que esta actividad física no se realiza de forma estructurada. Un hallazgo similar se evidencia en el riesgo de mortalidad de origen cardiovascular.

Ya evaluando programas de ejercicio estructurados, los hallazgos demostraron que 
tanto el ejercicio aeróbico como el ejercicio de resistencia impactan significativamente; pero es moderado en las concentraciones de HbA1c. Los datos disponibles sugieren que la combinación de ambos tipos de ejercicio produce un impacto similar. Adicionalmente, este impacto es mayor en la medida en que aumenta el número de sesiones semanales.

Existe suficiente evidencia a favor de iniciar manejo farmacológico con terapia combinada desde el inicio en pacientes con DMT2 recién diagnosticada, cuando en el momento del diagnóstico los valores de HbA1c son mayores del $8 \%$. Dada la similitud entre la eficacia de los medicamentos, la decisión de cuál utilizar debe basarse fundamentalmente en el perfil de riesgo asociado a cada uno.

La evidencia presentada sugiere que el mejor balance de riesgo beneficio está a favor de la terapia combinada entre metformina y DPP4, que son opciones razonables a la combinación entre metformina y sulfonilureas con bajo riesgo de hipoglucemia (glimepirida o glicazida), o la combinación entre metformina y SGLT2.

Con base en la información presentada en este artículo, el grupo desarrollador de la guía planteó las siguientes recomendaciones. De igual forma, la información se resume en los algoritmos de manejo (figuras 1 y 2 ).

Figura 1. Manejo inicial de pacientes con diabetes mellitus tipo 2

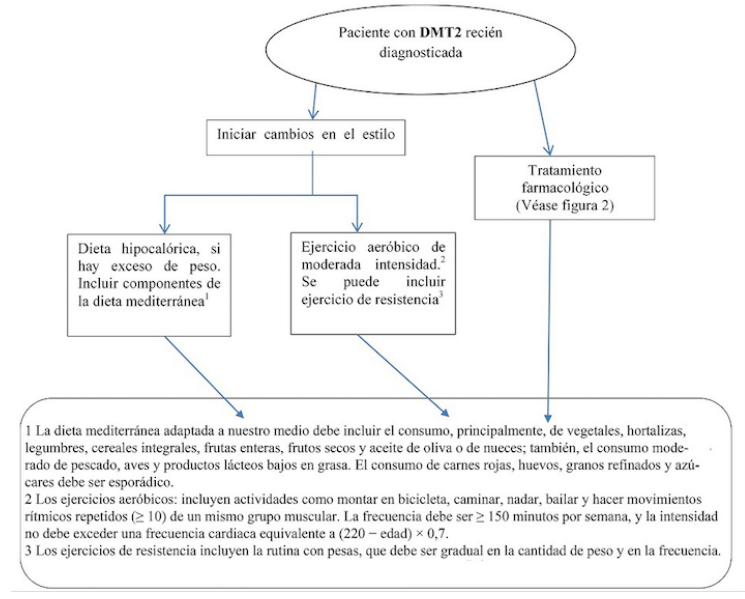

Figura 2. Manejo farmacológico inicial

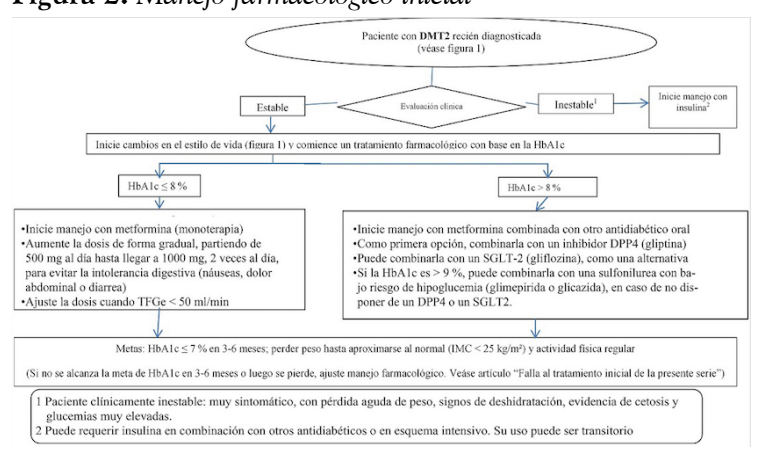

\section{Recomendaciones}

1. No se recomienda como tratamiento inicial en pacientes con DMT2 recién diagnosticada; el manejo únicamente debe ser con cambios en el estilo de vida. Recomendación fuerte en contra de la intervención. Calidad de la evidencia moderada

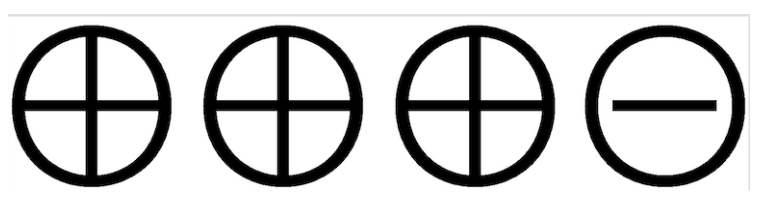

2. Se recomienda en pacientes con DMT2 recién diagnosticada; inicie el tratamiento farmacológico con metformina de forma simultánea con los cambios en el estilo de vida, aunque el valor inicial de la $\mathrm{HbA1c}$ esté cercano a la meta. Recomendación fuerte a favor de la intervención. Calidad de la evidencia moderada

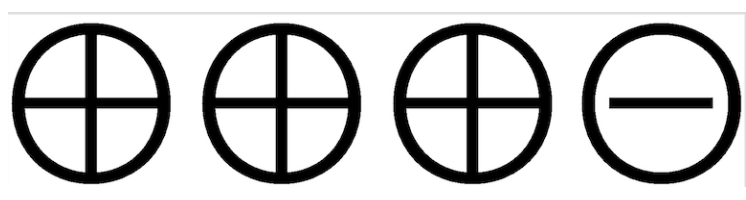

3. Se sugiere, en pacientes con DMT2 recién diagnosticada, que los cambios en el estilo de vida incluyan los componentes de la dieta mediterránea. Recomendación débil 
a favor. Calidad de la evidencia moderada

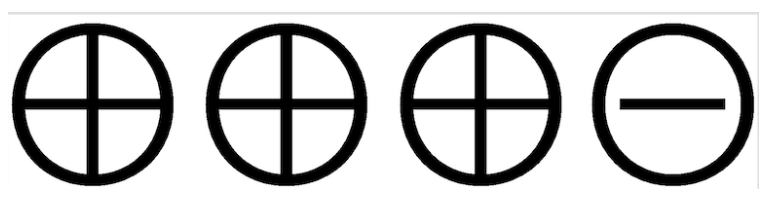

4. Se sugiere, en pacientes con DMT2 recién diagnosticada, que los cambios en el estilo de vida incluyan ejercicio aeróbico de moderada intensidad. Recomendación débil a favor. Calidad de la evidencia baja

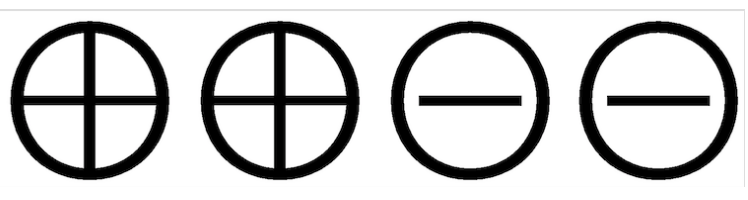

5. Se sugiere, en pacientes con DMT2 recién diagnosticada, que los cambios en el estilo de vida incluyan ejercicio de resistencia, en los casos en los que se haga manifiesta esta preferencia. Recomendación débil a favor. Calidad de la evidencia muy baja

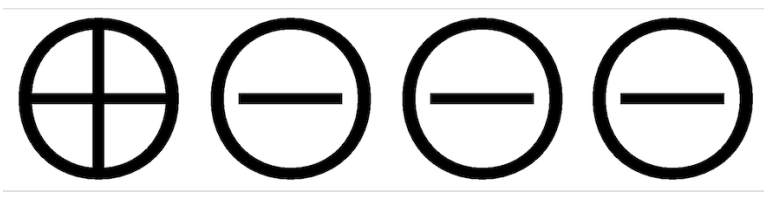

Puntos de buena práctica clínica

Cuando se inicie el cambio de estilo de vida se deben tener en cuenta los siguientes elementos:

Se recomienda adaptar la dieta mediterránea a las características del medio, pero conservando el predominio del consumo de vegetales, hortalizas, legumbres, cereales integrales, frutas enteras, frutos secos y aceite de oliva o nueces. Incluye consumo moderado de pescado, aves, productos lácteos bajos en grasa y vino con las comidas, si forma parte del hábito del paciente. El consumo de carnes rojas, huevos y granos refinados debe ser esporádico.

El consumo habitual de alcohol no se debe estimular.

Se debe establecer un programa estructurado de ejercicio para que este tenga un impacto favorable.

El ejercicio aeróbico incluye actividades como montar en bicicleta, caminar, nadar, bailar y movimientos rítmicos repetidos $(\geq 10)$ de un mismo grupo muscular. La frecuencia debe ser igual o mayor a 150 minutos por semana y la intensidad no debe exceder una frecuencia cardiaca equivalente a $(220-$ edad $) \times 0,7$.

$\mathrm{El}$ ejercicio de resistencia incluye rutina con pesas que debe ser gradual en la cantidad de peso y la frecuencia.

Se sugiere, en pacientes con DMT2 recién diagnosticada que tengan limitaciones físicas que le impidan la movilidad, individualizar la prescripción de ejercicio y que sea valorado por un fisiatra o un médico del deporte.

Si el paciente está clínicamente inestable, es preferible aplazar el comienzo del ejercicio hasta que esté compensado clínicamente.

La metformina debe dosificarse de forma gradual partiendo de $500 \mathrm{mg}$ al día hasta llegar a $1000 \mathrm{mg}$, dos veces al día, para evitar intolerancia digestiva (náuseas, dolor abdominal y diarrea).

Se debe reducir la dosis de metformina a un máximo de $1000 \mathrm{mg} /$ día cuando la tasa de filtración glomerular esté por debajo de $50 \mathrm{ml} /$ min y se debe suspender cuando esté por debajo de $30 \mathrm{ml} / \mathrm{min}$. Está contraindicada también cuando hay un riesgo alto de acidosis láctica, como en estados de hipoxemia severa, falla hepática y alcoholismo.

Cuando la metformina está contraindicada o no se tolera, se puede remplazar como tratamiento inicial por cualquiera de los otros antidiabéticos orales que esté aprobado para monoterapia.

6. En los pacientes con DMT2 recién diagnosticada y concentraciones de $\mathrm{HbA1C}$ 
mayores al $8 \%$, se recomienda utilizar terapia combinada desde el inicio con metformina y otro antidiabético oral. Recomendación fuerte a favor. Calidad de la evidencia moderada
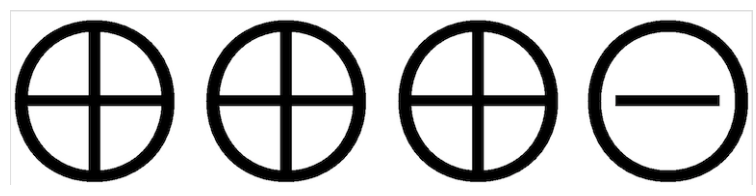

7. En los pacientes con DMT2 recién diagnosticada en quienes se decida implementar terapia combinada desde el inicio, se recomienda la asociación de metformina con un inhibidor de DPP-4. Recomendación fuerte a favor. Calidad de la evidencia Moderada
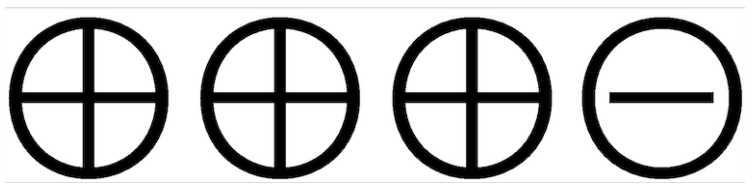

8. En los pacientes con DMT2 recién diagnosticada, en quienes se decida terapia combinada desde el inicio, se sugiere la combinación de metformina con un inhibidor SGLT-2, como una alternativa a la combinación de metformina con inhibidor DPP-4. Recomendación débil a favor. Calidad de la evidencia Moderada
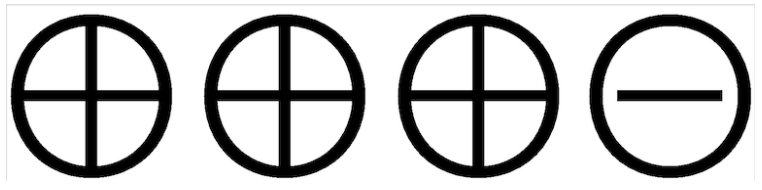

9. En los pacientes con DMT2 recién diagnosticada y la HbA1c mayor al $9 \%$ que no puedan utilizar la combinación de metformina con DPP4 o SGLT2, se sugiere la combinación de metformina con una sulfonilurea que tenga bajo riesgo de hipoglucemia (glimepirida o glicazida). Recomendación débil a favor. Calidad de la evidencia Moderada

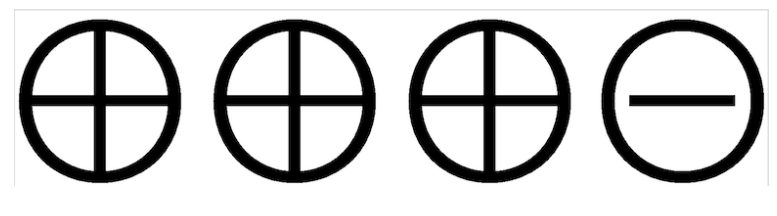

10. En los pacientes con DMT2 recién diagnosticada se sugiere no usar la combinación de metformina con glibenclamida, por el alto riesgo de hipoglucemia. Recomendación débil en contra. Calidad de la evidencia Moderada

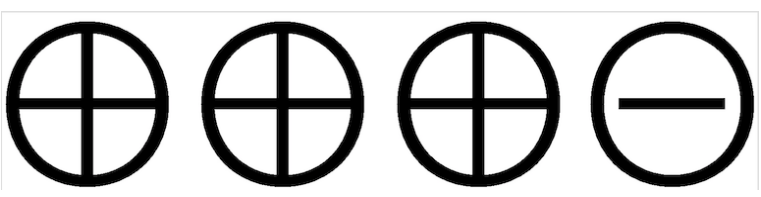

11. En los pacientes con DMT2 recién diagnosticada se sugiere no usar la combinación de metformina con tiazolidinedionas, por el riesgo aumentado de desarrollar edemas, falla cardiaca o fracturas. Recomendación fuerte en contra. Calidad de la evidencia Moderada

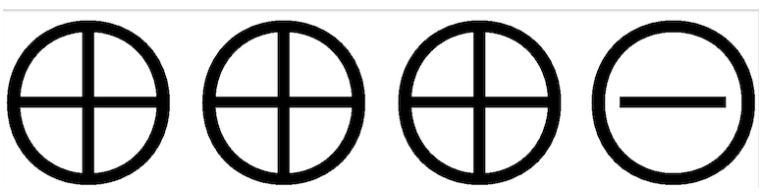

Puntos de buena práctica clínica

Se deben preferir las combinaciones fijas de metformina con otro antidiabético oral, siempre que estén disponibles, porque mejoran la adherencia.

La dosis de los inhibidores DPP4 debe ajustarse cuando la tasa de filtración glomerular descienda 
por debajo de $50 \mathrm{ml} / \mathrm{min}$, con excepción de linagliptina.

Se recomienda en pacientes con DMT2, en quienes se decida el uso de sulfonilureas, hacer hincapié en la educación y realizar auto monitoreo de glucosa para detectar y tratar apropiadamente los episodios de hipoglucemia.

No se recomienda usar sulfonilureas cuando la tasa de filtración glomerular descienda por debajo de $30 \mathrm{ml} / \mathrm{min}$, con excepción de glipizida.

Se recomienda vigilar y tratar oportunamente las infecciones genitourinarias cuando se usan los inhibidores SGLT2. Su eficacia tiende a disminuir a medida que la función renal se deteriora significativamente.

Si se presentan hipoglucemias con el uso de sulfonilureas, se debe considerar el cambio a medicamentos que no causen hipoglucemia.

\section{Agradecimientos}

Fuente de financiación Ministerio de Salud y Protección Social. Alianza Cinets. Agradecemos la contribución de las personas que, en carácter de representantes de expertos temáticos, usuarios, población blanco o grupos de interés, asistieron o participaron en las reuniones de socialización realizadas durante el desarrollo de la presente guía. Así mismo, a la Asociación Colombiana de Endocrinología, Diabetes y Metabolismo y la Asociación Colombiana de Diabetes.

\section{Referencias}

1. United Kingdom Prospective Diabetes Study. Intensive blood-glucose control with sulphonylureas or insulin compared with conventional treatment and risk of complications in patients with type 2 diabetes (UKPDS 33). Lancet. 1998;352 (Ukpds 33):837-53.

2. Prospective Diabetes Study Group U. Effect of intensive blood-glucose control with metformin on complications in overweight patients with type 2 diabetes. Lancet. 1998;352(Ukpds 34):854-65.
3. Canadian Diabetes Association Clinical Practice Guidelines Expert Committee, Cheng AY. Canadian Diabetes Asociation 2013 clinical practice guideline for the prevention and management of diabetes in Canada. Can J Diabetes. 2013;37 Suppl $1: S 1-3$.

4. Handelsman Y, Mechanick JI, Dagogo-jack S, Davidson JA. AACE Guidelines. American Association of Clinical Endocrinologist medical guidelines for clinical practice for devoloping a diabetes mellitus comprehensive care plan. Diabetes Care. 2011;1-53.

5. Asociación Latinoamericana de Diabetes. Guías ALAD 2013 para el diagnóstico y manejo de la diabetes mellitus tipo 2 con medicina basada en evidencia. Revista de la ALAD. 2013 [internet]. Disponible en: http://www.revistaalad.com/pdfs/Guias _ALAD_11_Nov_2013.pdf

6. Goldenberg $\overline{\mathrm{R}}$, Punthakee Z. Definition, classification and diagnosis of diabetes, prediabetes and metabolic syndrome. Can J Diabetes. 2013 Apr;37 Suppl 1:S8-11.

7. Giacco R, Vetrani C, Griffo E, Rivellese A. Role of diet and diet interventions in diabetic patients: physiological and metabolic changes and reduction in morbidity and mortality. Curr Nutr Rep. 2013 Oct;2(4):174-80.

8. Franz MJ, Powers MA, Leontos C, Holzmeister LA, Kulkarni K, Monk A, et al. The evidence for medical nutrition therapy for type 1 and type 2 diabetes in adults. J Am Diet Assoc. 2010 Dec;110(12):1852-89.

9. Estruch R, Ros E, Salas-Salvadó J, Covas M-I, Corella D, Arós F, et al. Primary prevention of cardiovascular disease with a Mediterranean diet. N Engl J Med. 2013 Apr 4;368(14):1279-90.

10. Sigal RJ, Armstrong MJ, Colby P, Kenny GP, Plotnikoff RC, Reichert SM, et al. Physical activity and diabetes. Can J Diabetes. 2013 Apr;37 Suppl 1:S40-4.

11. Nielsen PJ, Hafdahl AR, Conn VS, LeMaster JW, Brown SA. Meta-analysis of the effect of exercise interventions on fitness 
outcomes among adults with type 1 and type 2 diabetes. Diabetes Res Clin Pract. 2006;74(2):111-20.

12. Gordon BA, Benson AC, Bird SR, Fraser SF. Resistance training improves metabolic health in type 2 diabetes: A systematic review. Diabetes Res Clin Pract. 2009;83(2):157-75.

13. Andrews R, Cooper AR, Montgomery AA, Norcross AJ, Peters TJ, Sharp DJ, et al. Diet or diet plus physical activity versus usual care in patients with newly diagnosed type 2 diabetes: The Early ACTID randomised controlled trial2225. Lancet. 2011 Jul;378(9786):129-39.

14. American Diabetes Association. Standards of medical care in diabetes--2013. Diabetes Care. 2013 Jan;36 Suppl 1(October 2012):S11-66.

15. Phung OJ, Sobieraj DM, Engel SS, Rajpathak SN. Early combination therapy for the treatment of type 2 diabetes mellitus: systematic review and metaanalysis. Diabetes Obes Metab. 2014 May;16(5):410-7.

16. Zinman B. Initial combination therapy for type 2 diabetes mellitus: is it ready for prime time? Am J Med. 2011 Jan;124(1 Suppl):S19-34.

17. Garber a J, Larsen J, Schneider SH, Piper B a, Henry D. Simultaneous glyburide/metformin therapy is superior to component monotherapy as an initial pharmacological treatment for type 2 diabetes. Diabetes Obes Metab. 2002 May;4(3):201-8.

18. Rosenstock J, Goldstein BJ, Vinik AI, O'neill MC, Porter LE, Heise MA, et al. Effect of early addition of rosiglitazone to sulphonylurea therapy in older type 2 diabetes patients ( $>60$ years): the Rosiglitazone Early vs. SULphonylurea Titration (RESULT) study. Diabetes Obes Metab. 2006 Jan;8(1):49-57.

19. Rosenstock J, Rood J, Cobitz A, Biswas $\mathrm{N}$, Chou $\mathrm{H}$, Garber A. Initial treatment with rosiglitazone/metformin fixed-dose combination therapy compared with monotherapy with either rosiglitazone or metformin in patients with uncontrolled type 2 diabetes. Diabetes Obes Metab. 2006;8:650-60.

20. Shah BR, Hux JE, Laupacis A, Zinman B, van Walraven C. Clinical inertia in response to inadequate glycemic control. Diabetes Care. 2005 Mar;28(3):600-6.

21. Carrasquilla G, Pulido A, Mieth K, Muñoz O. Guía metodológica para la elaboración de guías de práctica clínica con evaluación económica en el Sistema General de Seguridad Social en Salud colombiano. Bogotá: Ministerio de Salud y Protección Social; 2014.

22. Wing RR, Bolin P, Brancati FL, Bray G a, Clark JM, Coday M, et al. Cardiovascular effects of intensive lifestyle intervention in type 2 diabetes. N Engl J Med. 2013 Jul $11 ; 369(2): 145-54$.

23. Yang B, Chen Y, Xu T, Yu Y, Huang T, Hu X, et al. Systematic review and meta-analysis of soy products consumption in patients with type 2 diabetes mellitus. Asia Pac J Clin Nutr. 2011 Jan;20(4):593-602.

24. Nield L, Moore H, Hooper L, Cruickshank K, Vyas A, Whittaker V, et al. Dietary advice for treatment of type 2 diabetes mellitus in adults (Review). Cochrane Database Syst Rev. 2009; (3):1-82.

25. National Collaborating Centre for Chronic Conditions. Type 2 diabetes: national clinical guideline for management in primary and secondary care (update). London: Royal College of Physicians; 2008.

26. Ajala O, English P, Pinkney J. Systematic review and meta-analysis of different dietary approaches to the management of type 2 diabetes 1-3. Am J Clin Nutr. 2013;97(3):505-16.

27. Thomas D, Elliott EJ. Low glycaemic index, or low glycaemic load, diets for diabetes mellitus. Cochrane Database Syst Rev. 2009;21(1)CD006296.

28. Sluik D, Buijsse B, Muckelbauer R, Kaaks R, Teucher B, Johnsen NF, et al. Physical activity and mortality in individuals with diabetes mellitus: a prospective study and 
meta-analysis. Arch Intern Med. 2012 Sep 24;172(17):1285-95.

29. Umpierre D, Kramer CK, Leita CB, Gross JL, Ribeiro JP, Schaan BD. Physical activity advice only or structured with $\mathrm{HbA}$ 1c levels in type 2 diabetes. JAMA. 2014;305:1790-9.

30. Umpierre D, Ribeiro PAB, Schaan BD, Ribeiro JP. Volume of supervised exercise training impacts glycaemic control in patients with type 2 diabetes: a systematic review with meta-regression analysis. Diabetologia. 2013 Feb;56(2):242-51.

31. Patil HR, Al Badarin FJ, Al Shami HA, Bhatti SK, Lavie CJ, Bell DSH, et al. Metaanalysis of effect of dipeptidyl peptidase-4 inhibitors on cardiovascular risk in type 2 diabetes mellitus. Am J Cardiol. 2012 Sep 15;110(6):826-33.

32. Kong Y, Shing K. Comparative cardiovascular effects of thiazolidinediones: systematic review and meta-analysis of observational studies. BMJ. 2011 Jan;342:d1309.

33. Wu D, Li L, Liu C. Efficacy and safety of dipeptidyl peptidase-4 inhibitors and metformin as initial combination therapy and as monotherapy in patients with type 2 diabetes mellitus: a meta-analysis. Diabetes Obes Metab. 2014 Jan;16(1):30-7.

34. Perez A, Zhao Z, Jacks R, Spanheimer R. Efficacy and safety of pioglitazone/ metformin fixed-dose combination therapy compared with pioglitazone and metformin monotherapy in treating patients with T2DM. Curr Med Res Opin. 2009;25:2915-23.

35. Henry RR, Murray A V, Marmolejo MH, Hennicken D, Ptaszynska A, List JF. Dapagliflozin, metformin XR, or both\#: initial pharmacotherapy for type 2 diabetes, a randomised controlled trial. Int J Clin Pr. 2012;66(May):446-56.

36. Zhang F, Xiang H, Fan Y, Ganchuluun T-A, Kong W, Ouyang Q, et al. The effects of sulfonylureas plus metformin on lipids, blood pressure, and adverse events in type 2 diabetes: A meta-analysis of randomized controlled trials. Endocrine. 2013 Dec;44(3):648-58.

37. Lincoff A, Wolski K, Nicholls SJ, Nissen SE. Pioglitazone and risk of cardiovascular events in patients with type 2 diabetes mellitus. JAMA. 2014;298(10):1180-8.

38. Loke Y, Singh S, Furberg C. Long-term use of thiazolidinediones and fractures in type 2 diabetes: a meta-analysis. CMAJ. 2009;180(1):32-9.

39. Ferwana M, Firwana B, Hasan R, AlMallah MH, Kim S, Montori VM, et al. Pioglitazone and risk of bladder cancer: a meta-analysis of controlled studies. Diabet Med. 2013 Sep;30(9):1026-32. 